\title{
Effect of exercise intensity on postexercise energy expenditure in women*
}

\author{
D. A. Sedlock PhD \\ Exercise Physiology Laboratory, Purdue University, West Lafayette, Indiana, USA
}

\begin{abstract}
This study was designed to examine the effect of exercise intensity on the magnitude and duration of excess postexercise oxygen consumption (EPOC) in women. On separate days and in a counterbalanced order, seven moderately active young adult women performed a $850 \mathrm{~kJ}$ cycle ergometer exercise at an intensity of 40 or $60 \%$ of their previously determined peak oxygen uptake $\left(\dot{V}_{\mathrm{O}_{2}}\right)$. Baseline $\dot{V}_{2}$ and heart rate (HR) were recorded during the last $10 \mathrm{~min}$ of a $45 \mathrm{~min}$ seated rest. $\dot{V} \mathrm{O}_{2}$ and $\mathrm{HR}$ were measured continuously during recovery until $\dot{V}_{\mathrm{O}_{2}}$ returned to baseline. There was no significant difference noted in the baseline measures between the two exercise programmes. Magnitude of EPOC was comparable $(P>0.05)$ being mean (s.d.) of 30(17) and 36(13) kJ after 40 and $60 \%$ exercises respectively. Although the EPOC duration was $53 \%$ longer following the $40 \%$ exercise than following $60 \%(27(15) \mathrm{min}$ and $18(8) \mathrm{min}$, respectively) this difference was not statistically significant. These exercise conditions failed to produce a prolonged EPOC in the women of this study, and values recorded for magnitude of EPOC indicate that it was not significant with regard to the overall energy expenditure of the activity. It was concluded that both magnitude and duration of EPOC seemed to be independent of the chosen exercise intensities used by the women in this study.
\end{abstract}

Keywords: Recovery metabolism, women, cycle ergometer

One important factor that must be addressed when prescribing exercise is that the programme be designed to meet the specific objectives of the client. Evidence indicates, for example, that many women engage in aerobic exercise to reduce or control their body weight ${ }^{1}$. In order to develop the most effective exercise for this particular group, professionals must know how to maximize energy expenditure among women, and be aware of their metabolic responses to exercise.

Energy expenditure associated with physical activity includes that expended during the exercise itself and that consumed in the postexercise or recovery period. Although results reported in the literature concerning the excess postexercise oxygen consump-

* This study was supported by a XL grant from the Purdue Research Foundation

Address for correspondence: $\mathrm{Dr} \mathrm{D}$. A. Sedlock PhD, Exercise Physiology Laboratory, Department of PEHRS, Lambert 007, Purdue University, West Lafayette, IN 47097, USA

(C) 1991 Butterworth-Heinemann Ltd.

0306-3674/91/010038-03 tion (EPOC) are equivocal as to both the magnitude and duration of this period ${ }^{2-7}$, it has been reported that postexercise energy expenditure may be influenced by manipulating the intensity ${ }^{3,7}$ or duration ${ }^{8}$ of isocaloric exercise. However, our understanding of the overall EPOC response is largely confined to men since many EPOC studies have included male subjects $2,4,5,7,9-11$, whereas only one study was found that involved women exclusively ${ }^{12}$.

The purpose of this study was therefore to examine postexercise energy expenditure in women. Investigations of EPOC duration are critical in attempting to identify the underlying physiological mechanisms, whereas quantifying the consequent magnitude has the greater impact regarding weight control. It has recently been shown that these two issues are not necessarily related, i.e. a longer EPOC duration does not necessarily result in a greater energy expenditure ${ }^{7}$, suggesting that each of these should be addressed independently. Therefore, this study specifically investigated the effect of exercise intensity on both magnitude and duration of EPOC with exercise energy expenditure held constant.

\section{Methods}

Subjects. Seven moderately trained women volunteered for the study. Mean (s.d.) age, height, and weight were $25.7(4.0)$ years, $165.8(5.2) \mathrm{cm}$, and $61.3(5.9) \mathrm{kg}$, respectively. All were physically active at the time of the study, although none was engaged in any systematic training. The procedures used in this investigation were reviewed and approved by the institutional committee for research involving human subjects. All subjects expressed their understanding of the procedures and their rights as subjects by signing a statement of informed consent.

Protocol. Subjects performed two submaximal cycle ergometer exercises that elicited an energy expenditure of $850 \mathrm{~kJ}$. One was at an intensity of $40 \%$ and the other at $60 \%$ of each subject's previously determined peak oxygen uptake $\left(\dot{V}_{\mathrm{O}_{2}}\right)$. The exercise tests were administered in a counterbalanced order and were separated by a minimum of 2 days.

Test for peak $\dot{V}_{2}$. A continuous, incremental exercise test was performed using a mechanically-braked cycle ergometer to determine peak $\dot{V}_{\mathrm{O}_{2}}$. Initial power output (PO) was $60 \mathrm{~W}$ at 60 r.p.m., with subsequent 
Postexercise energy expenditure: D. A. Sedlock

increases of $30 \mathrm{~W}$ every $2 \mathrm{~min}$ until termination of the test. Criteria for termination of the test were: (1) an increase in $\mathrm{PO}$ with little or no increase in $\mathrm{VO}_{2} ;$ (2) heart rate (HR) at or near the age-predicted maximum; or (3) failure to maintain the prescribed cranking rate due to fatigue. The highest minute value obtained for $\dot{V}_{\mathrm{O}_{2}}$ was recorded as peak $\dot{V}_{2}$.

Submaximal exercise test. Subjects reported to the laboratory at approximately 6.00 hours following an overnight fast and having refrained from any strenuous physical activity for 24-36h. After applying surface electrodes to monitor $\mathrm{HR}$, the subjects sat quietly in a chair for $45 \mathrm{~min}$. Baseline $\mathrm{VO}_{2}$ and $\mathrm{HR}$ were measured during the last $15 \mathrm{~min}$ of this period. The exercise was then performed at the prescribed intensity, with subjects again seated quietly in a chair immediately after termination of the exercise. $\dot{V O}_{2}$ and $\mathrm{HR}$ were monitored continuously during the recovery period until $\dot{V}_{\mathrm{O}_{2}}$ returned to the baseline value.

Measurements. $\quad \dot{\mathrm{V}} \mathrm{O}_{2}$ was measured by open circuit spirometry. Subjects inspired room air, and expired air was metered and analysed for fractional oxygen and carbon dioxide using an automated system (Quinton Q-Plex I, Seattle, WA, USA). $\dot{V}_{2}$ was converted to energy expenditure (kJ) using the caloric equivalent of 1 litre of oxygen based on the non-protein respiratory exchange ratio (RER).

Baseline $\dot{V}_{\mathrm{O}_{2}}$ and $\mathrm{HR}$ were determined by averaging the minute values for these variables obtained during the last $10 \mathrm{~min}$ of the $15 \mathrm{~min}$ baseline measurement period. Duration of EPOC was determined by continuously averaging five consecutive $1 \mathrm{~min}$ values of $\dot{\mathrm{O}}_{2}$ during the postexercise period. When this average was equal to the baseline value, duration was recorded as the elapsed time ( $\mathrm{min}$ ) from termination of the exercise to the first minute of this $5 \mathrm{~min}$ average. Magnitude of EPOC was obtained by summing the net energy expenditure for each minute of the EPOC period.

\section{Results}

Mean baseline values obtained before exercise at 40 and $60 \%$ of peak $\dot{V}_{2}$ are shown in Table 1 . No significant difference was noted for any of the variables, indicating that the subjects were in a comparable resting state before both experiments.

Table 2 includes data obtained during the exercises. The target intensities were 40 and $60 \%$ peak $\dot{V}_{\mathrm{O}_{2}}$. Actual values were 41.1 and $61.8 \%$, respectively. As expected, a significantly higher $\dot{V} \mathrm{O}_{2}(t=22.6, P<$ $0.01)$ and HR $(t=10.1, P<0.01)$ response was elicited during the $60 \%$ exercise compared with the $40 \%$ exercise. Energy expenditure for the two exercises was similar, and therefore required a significantly longer duration $(t=-21.8, P<0.01)$ when exercising at $40 \%$ peak $\dot{\mathrm{V}}_{2}$.

Data obtained during the EPOC period are shown in Table 3. There was no significant difference in duration of EPOC; however, the mean value following exercise at $40 \%$ peak $\dot{\mathrm{O}}_{2}$ was $53 \%$ longer than
Table 1. Mean (s.d.) values measured during the baseline period

\begin{tabular}{|c|c|c|c|}
\hline Variable & $\begin{array}{c}\text { Before } 40 \% \\
\text { exercise }\end{array}$ & $\begin{array}{c}\text { Before } 60 \% \\
\text { exercise }\end{array}$ & $t^{*}$ \\
\hline 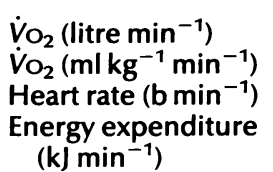 & $\begin{array}{l}0.22(0.03) \\
3.67(0.45) \\
62(6) \\
4.62(0.59)\end{array}$ & $\begin{array}{c}0.23(0.03) \\
3.68(0.20) \\
59(5) \\
\\
4.70(0.59)\end{array}$ & $\begin{array}{r}0.21 \\
0.13 \\
-1.38 \\
\\
0.51\end{array}$ \\
\hline
\end{tabular}

$* P>0.05$

Table 2. Mean (s.d.) values obtained during the two exercises

\begin{tabular}{|c|c|c|c|}
\hline Variable & $\begin{array}{c}40 \% \text { peak } \\
\dot{\mathrm{VO}_{2}}\end{array}$ & $\begin{array}{c}60 \% \text { peak } \\
\dot{V}_{\mathrm{O}_{2}}\end{array}$ & $t$ \\
\hline $\begin{array}{l}\% \text { Peak } \dot{V}_{\mathrm{O}_{2}} \\
\dot{V} \mathrm{O}_{2}\left(\text { litre } \min ^{-1}\right) \\
\text { Heart rate }\left(\mathrm{b} \mathrm{min}^{-1}\right) \\
\text { Energy expenditure (kJ) } \\
\text { Duration (min) }\end{array}$ & $\begin{array}{c}41.1(0.89) \\
1.03(0.13) \\
108(9) \\
871.1(26.0) \\
41.1(5.2)\end{array}$ & $\begin{array}{c}61.8(1.83) \\
1.54(0.19) \\
137(3) \\
879.5(31.9) \\
27.3(3.6)\end{array}$ & $\begin{array}{r}36.92^{*} \\
22.61^{*} \\
10.16^{*} \\
1.70 \\
-21.87^{*}\end{array}$ \\
\hline
\end{tabular}

$* P<0.05$

Table 3. Mean (s.d.) values measured during the postexercise period for the two experiments

\begin{tabular}{|c|c|c|c|}
\hline Variable & $\begin{array}{c}\text { After } 40 \% \\
\text { exercise }\end{array}$ & $\begin{array}{c}\text { After } 60 \% \\
\text { exercise }\end{array}$ & $t^{*}$ \\
\hline $\begin{array}{l}\text { Duration }(\min ) \\
\text { EPOC }(k J) \\
\text { Heart rate }\left(b \min ^{-1}\right)+\end{array}$ & $\begin{array}{l}27.7(15.5) \\
29.8(17.2) \\
62(4)\end{array}$ & $\begin{array}{l}18.1(8.4) \\
36.1(12.6) \\
67(5)\end{array}$ & $\begin{array}{r}-1.65 \\
0.78 \\
2.25\end{array}$ \\
\hline
\end{tabular}

$* P>0.05$

+ Heart rate recorded at the end of the period of excess postexercise oxygen consumption. Value following exercise at $60 \%$ peak $\dot{\mathrm{O}}_{2}$ was significantly higher $(P<0.05)$ than baseline.

the EPOC duration following the $60 \%$ exercise. This lack of statistical significance was probably due to the relatively large interindividual variation recorded for both conditions. On the other hand, magnitude of EPOC was similar following the two exercises.

Although there was no significant difference in mean HR measured at the end of the EPOC period between the two conditions $(P<0.07)$, end-EPOC HR was significantly elevated $(P<0.05)$ above baseline following the $60 \%$ exercise.

\section{Discussion}

This investigation was designed to examine the effect of exercise intensity on postexercise energy expenditure in women when exercise energy expenditure was held constant. Only one other EPOC study was located that included women as subjects ${ }^{12}$. However, that study compared the recovery response to exercise with and without a caffeine challenge, with the exercise performed at a constant intensity (55\% $\left.\dot{\mathrm{V}}_{2}, \max \right)$ and duration $(90 \mathrm{~min})$ for both experiments. 
Moreover, $\dot{V} \mathrm{O}_{2}$ was still elevated at the time the measurements were terminated. Therefore, neither magnitude nor duration of EPOC were reported. Results in this study indicate that a $850 \mathrm{~kJ}$ exercise at 40 and $60 \%$ peak $\mathrm{VO}_{2}$ had no differential effect on either the magnitude or duration of EPOC. Further, energy expenditure measured during the postexercise period was relatively low following both exercise experiments.

It is commonly believed that the elevated metabolic rate during recovery from exercise exists for several hours. This tenet is based on several reports of this phenomenon spanning 50 years ${ }^{2,4,9,13,14}$. In contrast to those studies, which included males as subjects, the results of this study using women did not show a prolonged elevated metabolic rate during recovery from exercise.

Although a prolonged EPOC has been demonstrated in the above-mentioned studies, results of other investigations have not supported this finding $3,5,7,15$. Reasons for these discrepant findings are unclear, although exercise intensity and duration have been suggested as a possibility ${ }^{16}$. With regard to exercise intensity, Brehm and Gutin ${ }^{3}$ have proposed a model for postexercise energy expenditure after exercise of moderate duration, such as that used in the present study. The authors suggest that recovery energy expenditure is only minimally affected when exercise intensity is within 'the low to moderate range (30-50\% $\mathrm{VO}_{2}$, max $)$. At higher exercise intensities, the slope of the curve may increase sharply' (Reference 3, page 209). Results of the present study on women do not seem to support this model. Since little is known about the EPOC response in women, it is difficult to speculate why this had occurred. However, there are several possibilities. First, the study of Brehm and Gutin ${ }^{3}$ included both males and females, suggesting that perhaps differences in the sex of the subject groups between the latter study and the present study may have influenced the results. Second, the subjects of Brehm and Gutin ${ }^{3}$ were relatively exercise-trained whereas subjects of the present study were not engaged in any systematic training. Third, although one of the exercise intensities used in this study was above and the other below the implied threshold value of $50 \% \dot{V} \mathrm{O}_{2}, \max$, it could be that they were not sufficiently different from this threshold to show the EPOC response.

Although no threshold value for duration of exercise has been identified as producing the EPOC response, evidence suggests that a minimum of $1 \mathrm{~h}$ of moderate intensity exercise is necessary to affect the duration of EPOC. For example, a prolonged EPOC period has been reported following exercise durations of $180 \mathrm{~min}^{9}, 150 \mathrm{~min}^{13}$, and $90 \mathrm{~min}^{6,12}$. Additionally, Sedlock et al. ${ }^{7}$ reported that a $60 \mathrm{~min}$ exercise gave a significantly longer EPOC than a $30 \mathrm{~min}$ exercise. In contrast, the exercise durations of the present study were less than 1 h (i.e. 27 and $41 \mathrm{~min}$ ) and resulted in relatively short EPOC durations that were not significantly different from each other.

In summary, this study employed exercise intensities that are similar to what is often used by women for purposes of weight control, i.e. $850 \mathrm{~kJ}$ exercise energy expenditure at 40 and $60 \%$ peak $\dot{V}_{2}$. These conditions failed to elicit a prolonged elevated metabolic rate during the postexercise period, and the magnitude of EPOC suggests that the postexercise energy expenditure was not significant in relation to the total energy expenditure. Moreover, with the energy cost of the exercise held constant, both magnitude and duration of EPOC seemed to be independent of exercise intensity.

\section{References}

1 Duda JL, Tappe MK. Personal investment in exercise among adults: the examination of age- and gender-related differences in motivational orientation. In: Ostrow, A. ed. Aging and Motor Behavior. Indianapolis; Benchmark Press, 1989: 239-56.

2 Bahr R, Ingnes I, Vaage O. Sejersted OM, Newsholme EA. Effect of duration of exercise on excess postexercise $\mathrm{O}_{2}$ consumption. J Appl Physiol 1987; 62: 485-90.

3 Brehm BA, Gutin B. Recovery energy expenditure for steady state exercise in runners and nonexercisers. Med Sci Sports Exerc 1986; 18: 205-10.

4 Edwards HT, Thorndike A, Dill DB. The energy requirement in strenuous muscular exercise. N Engl J Med 1935; 213: 532-5.

5 Kaminsky LA, Kanter MM, Lesmes GR, Laham-Saeger J. Excess oxygen consumption following exercise of different intensity and duration. Can I Sport Sci 1987; 12: 237-9.

6 Maehlum S, Grandmontagne M, Newsholme EA, Sejersted OM. Magnitude and duration of excess postexercise oxygen consumption in healthy young subjects. Metabolism 1986; 35: $425-9$.

7 Sedlock DA, Fissinger JA, Melby CL. Effect of exercise intensity and duration on postexercise energy expenditure. Med Sci Sports Exerc 1989; 21: 662-6.

8 Chad KE, Wenger HA. The effects of duration and intensity on the exercise and postexercise metabolic rate. Aust J Sci Med Sport 1985; 17: 14-18.

9 Bielinski R, Schutz Y, Jequier E. Energy metabolism during the postexercise recovery in man. Am J Clin Nutr 1985; 42: 69-82.

10 Gore CI, Withers RT. Effect of exercise intensity and duration on postexercise metabolism. J Appl Physiol 1990; 68: 2362-8.

11 Pivarnik JM, Wilkerson JE. Recovery metabolism and thermoregulation of endurance trained and heat acclimatized men. J Sports Med Phys Fitness 1988; 28: 375-80.

12 Chad K, Quigley B. The effects of substrate utilization, manipulated by caffeine, on post-exercise oxygen consumption in untrained female subjects. Eur J Appl Physiol 1989; 59: 48-54.

13 Passmore R, Johnson RE. Some metabolic changes following prolonged moderate exercise. Metabolism 1960; 9: 452-6.

14 deVries HA, Gray DE. After-effects of exercise upon metabolic rate. Res $Q$ 1963; 34: 315-21.

15 Freedman-Akabas S, Colt E, Kissileff HR, Pi-Sunyer FX. Lack of sustained increase in $\dot{V}_{\mathrm{O}_{2}}$ following exercise in fit and unfit subjects. Am J Clin Nutr 1985; 41: 545-9.

16 Brehm BA. Elevation of metabolic rate following exercise: implications for weight loss. Sports Med 1988; 6: 72-8. 